\title{
Lattice QCD with nonzero chiral chemical potential
}

\section{V. Braguta}

ITEP, Moscow, 117218 Russia

E-mail: braguta@itep.ru

\section{E.-M. Ilgenfritz}

JINR, Dubna, Moscow region, 141980 Russia

E-mail: ilgenfriatheor.jinr.ru

\section{A. Yu. Kotov*}

ITEP, Moscow, 117218 Russia

E-mail: kotoveitep.ru

\section{Müller-Preussker}

Humboldt-Universität zu Berlin, Institut für Physik, Berlin, 12489 Germany

E-mail: mmp@physik. hu-berlin.de

\section{A. Schreiber}

Humboldt-Universität zu Berlin, Institut für Physik, Berlin, 12489 Germany

E-mail: alexander.schreiber@physik.hu-berlin.de

\section{B. Petersson}

Humboldt-Universität zu Berlin, Institut für Physik, Berlin, 12489 Germany

E-mail: bengt@physik.hu-berlin.de

\begin{abstract}
The phase diagram of two-color QCD with a chiral chemical potential is studied on the lattice. The focus is on the confinement/deconfinement phase transition and the breaking/restoration of chiral symmetry. The simulations are carried out with dynamical staggered fermions without rooting. The dependence of the Polyakov line, the chiral condensate and the corresponding susceptibilities on the chiral chemical potential and the temperature are presented.
\end{abstract}

The 32nd International Symposium on Lattice Field Theory,

23-28 June, 2014

Columbia University New York, $N Y$

${ }^{*}$ Speaker. 


\section{Introduction}

It is well known that the vacuum state of QCD for zero temperature or the statistical ensemble of gauge fields in the non-zero temperature case have nontrivial topological structures due to the presence of instantons or calorons, respectively, i.e. gluon configurations with nonzero topological charge $[1,2,3]$. For a long time their existence was only indirectly confirmed by the solution of the famous $U_{A}(1)$ problem $[4,5]$.

Recently it was pointed out that the existence of topologically nontrivial gluon configurations may be observed in experiments on heavy ion collisions [6, 7]. At such experiments blobs of hot matter - quark-gluon plasma - are assumed to be created in the presence of an external magnetic field which is expected to be present in non-central collisions. Topological sphaleron transitions $[8,9,10]$ due to the chiral anomaly lead to a nonzero chiral density within the quark-gluon plasma. This chiral medium is characterized by different densities of left- and right-handed quarks. The presence of the external magnetic field leads to a nonzero electric current along the direction of the field - it is the essence of the so-called chiral magnetic effect (CME) [6, 7]. Some indirect consequences of this effect have been observed at heavy-ion collision experiments [11, 12]. For the understanding of the CME it is important to study the phase diagram of QCD. For instance, one can argue that it is crucial for this effect that QCD matter is in the deconfined chirally restored phase $[6,7]$.

In this study we concentrate on the question how such phase transitions in QCD as the onset of deconfinement and chiral restoration are affected by a nonzero chiral density. One of the possible ways to study QCD with nonzero chiral density are lattice simulations with a chiral chemical potential $\mu_{5}$. The latter creates a difference between densities of left- and right- handed quarks. Contrary to an ordinary chemical potential $\mu$, simulations with a chiral chemical potential are not hampered by a sign problem and can be performed by means of standard Monte-Carlo algorithms [7].

In our study we perform simulations with the $S U(2)$ gauge group. We consider $S U(2)$ instead of $S U(3)$ because these theories have similar physical properties while the $S U(2)$ theory requires less computational resources.

Lattice simulations of QCD with a nonzero chiral chemical potential were already performed in $[13,14]$. The main goal of these articles was the study of the CME, however, the phase diagram was not systematically studied. The phase diagram with nonzero chirality was already studied by means of effective models $[15,16,17,18,19]$ with which we will compare our results.

\section{Details of the simulations}

We have performed simulations with the $S U(2)$ gauge group. We employ the standard Wilson plaquette action

$$
S_{g}=\beta \sum_{x, \mu<v}\left(1-\frac{1}{N_{c}} \operatorname{Tr} U_{\mu v}(x)\right)
$$


For the fermionic part of the action we use staggered fermions

$$
\begin{aligned}
S_{f}=m a \sum_{x} \bar{\psi}_{x} \psi_{x} & +\frac{1}{2} \sum_{x \mu} \eta_{\mu}(x)\left(\bar{\psi}_{x+\mu} U_{\mu}(x) \psi_{x}-\bar{\psi}_{x} U_{\mu}^{\dagger}(x) \psi_{x+\mu}\right)+ \\
& +\frac{1}{2} \mu_{5} a \sum_{x} s(x)\left(\bar{\psi}_{x+\delta} \bar{U}_{x+\delta, x} \psi_{x}-\bar{\psi}_{x} \bar{U}_{x+\delta, x}^{\dagger} \psi_{x+\delta}\right),
\end{aligned}
$$

where the $\eta_{\mu}(x)$ are the standard staggered phase factors: $\eta_{1}(x)=1, \eta_{\mu}(x)=(-1)^{x_{1}+\ldots+x_{\mu-1}}$ for $\mu=2,3,4$. Furthermore, $a$ denotes the lattice spacing, $m$ the bare fermion mass, and $\mu_{5}$ the value of the chiral chemical potential. In the chirality breaking term $s(x)=(-1)^{x_{2}}, \delta=(1,1,1,0)$ represents a shift to a diagonally located site of a spatial elementary cube, $\bar{U}_{x+\delta, x}=\frac{1}{6} \sum_{i, j, k=\operatorname{perm}(1,2,3)} U_{i}\left(x+e_{j}+\right.$ $\left.e_{k}\right) U_{j}\left(x+e_{k}\right) U_{k}(x)$ is a gauge factor connecting sites $x$ and $x+\delta$ symmetrized over 6 shortest ways between these sites.

The chirality breaking term is introduced in such a way that it has a correct naive continuum limit $a \rightarrow 0$

$$
S_{f} \rightarrow S_{f}^{(\text {cont })}=\int d^{4} x \sum_{i=1}^{4} \bar{\psi}_{i}\left(\partial_{\mu} \gamma_{\mu}+i g A_{\mu} \gamma_{\mu}+m+\mu_{5} \gamma_{5} \gamma_{4}\right) \psi_{i}
$$

The factor $s(x)$ corresponds to the product of "staggered" $\gamma$ matrices.

$$
\gamma_{5} \gamma_{4}=\gamma_{1} \gamma_{2} \gamma_{3} \Rightarrow 1 *(-1)^{x_{1}} *(-1)^{x_{1}+x_{2}}=s(x)
$$

We have performed simulations with lattice size $N_{\tau} \times N_{\sigma}^{3}=6 \times 16^{3}$. The measured observables are:

- the Polyakov loop

$$
L=\frac{1}{N_{\sigma}^{3}} \sum_{n_{1}, n_{2}, n_{3}}\left\langle\operatorname{Tr} \prod_{n_{4}=1}^{N_{\tau}} U_{4}\left(n_{1}, n_{2}, n_{3}, n_{4}\right)\right\rangle
$$

- the chiral condensate

$$
a^{3}\langle\bar{\psi} \psi\rangle=-\frac{1}{N_{\tau} N_{\sigma}^{3}} \frac{1}{4} \frac{\partial}{\partial(m a)} \log Z=\frac{1}{N_{\tau} N_{\sigma}^{3}} \frac{1}{4}\left\langle\operatorname{Tr} \frac{1}{D+m a}\right\rangle
$$

- the Polyakov loop susceptibility

$$
\chi_{L}=N_{\sigma}^{3}\left(\left\langle L^{2}\right\rangle-\langle L\rangle^{2}\right)
$$

- the disconnected part of the chiral susceptibility

$$
\chi_{\text {disc }}=\frac{1}{N_{\tau} N_{\sigma}^{3}} \frac{1}{16}\left(\left\langle\left(\operatorname{Tr} \frac{1}{D+m a}\right)^{2}\right\rangle-\left\langle\operatorname{Tr} \frac{1}{D+m a}\right\rangle^{2}\right) .
$$

The Polyakov loop and the corresponding susceptibility are sensitive to the confinement/deconfinement phase transition, while the chiral condensate responds to chiral symmetry breaking/restoration.

The simulations have been carried out with a CUDA code to run Hybrid Monte Carlo algorithm on GPU's. The dependence of the lattice spacing on the coupling parameter $\beta$ is taken from [20]. The fermion mass is fixed in lattice units at $m a=0.01$. For $\beta=1.8$ it corresponds to a pion mass value $m_{\pi} \approx 330 \mathrm{MeV}$. 


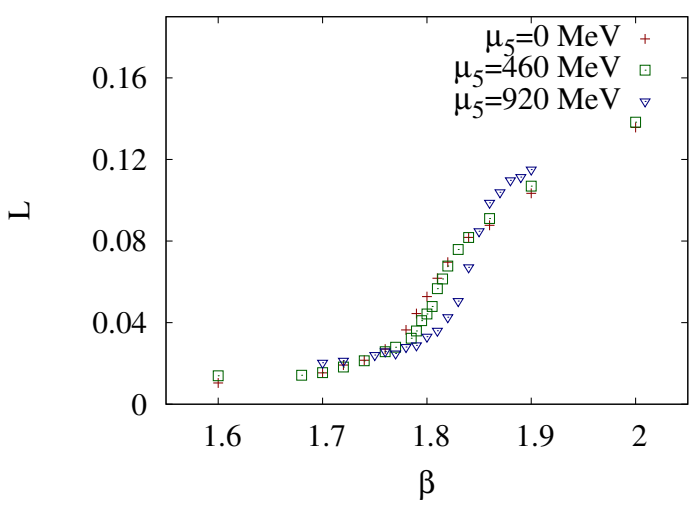

Polyakov loop

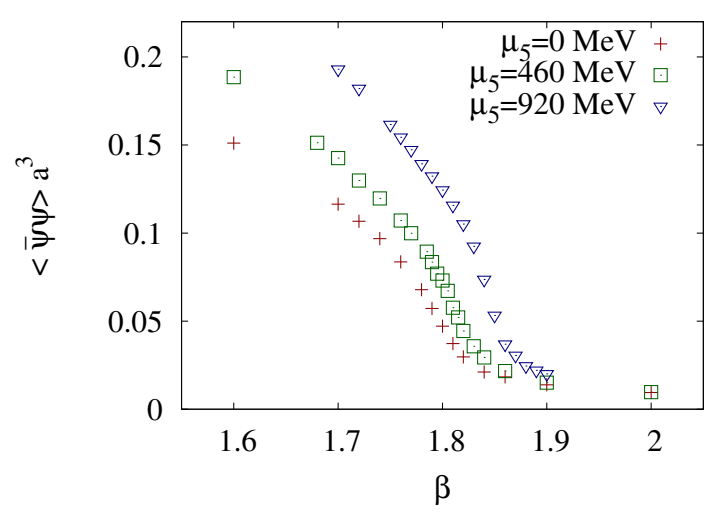

chiral condensate

Figure 1: Polyakov loop and chiral condensate versus $\beta$ for three values of the chiral chemical potential. Errors are smaller than the data points.

\section{Results and conclusions}

We present results for three fixed values of $\mu_{5}=0,460,920 \mathrm{MeV}$ and for different values of $\beta$, while the bare fermion mass remained constant in lattice units $m a=0.01$. The results for the Polyakov loop and the chiral condensate are plotted in Fig. 1. We see the increasing chiral chemical potential to move the position of the deconfinement and chiral transition, respectively, to larger values of $\beta$. This means that transition temperature increases. Plots for the chiral susceptibility and the Polyakov loop susceptibility (see Fig.2) confirm this observation. We estimate the change of the critical temperature to be $\frac{T_{c}\left(\mu_{5}\right)-T_{c}(0)}{T_{c}(0)} \sim 20 \%$ for $\mu_{5}=920 \mathrm{MeV}$. The results do not show any splitting between the chiral and deconfinement transitions.

Our results are in contradiction with those of the models studied in $[17,18,19]$, where the critical temperature of the transition was observed to decrease. Furthermore, in these papers at some critical value of the chiral chemical potential the transition was reported to become first order.

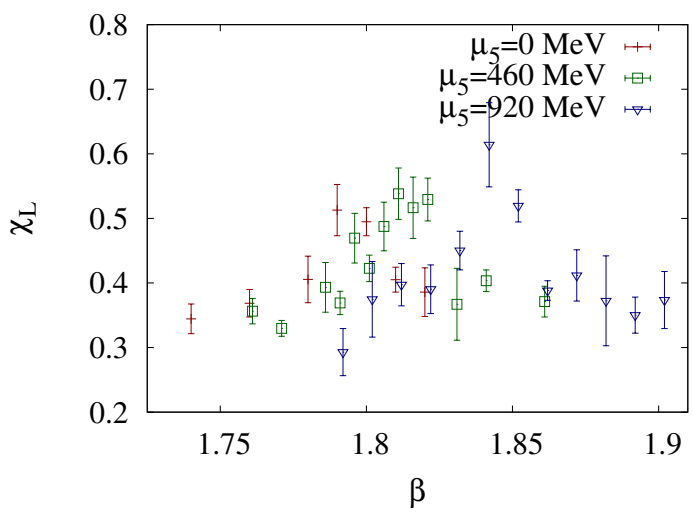

Polyakov loop susceptibility

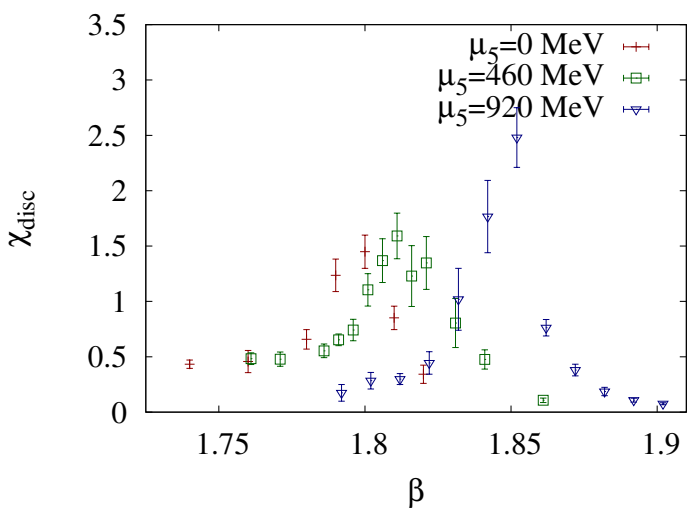

chiral susceptibility

Figure 2: Polyakov loop susceptibility and chiral susceptibility versus $\beta$ for three values of $\mu_{5}$. In order to avoid a complete superposition of data points belonging to different $\mu_{5}$ values we applied a tiny shift along the $\beta$ axis. 
In our simulations we do not see such a behavior. However, the results obtained have a tendency towards a sharper phase transition at nonzero chiral chemical potential. It is worth mentioning that the behavior described above looks quite similar as that obtained for two-color QCD in an external magnetic field [20,21].

Of course, it should be noted that we use the $S U(2)$ gauge group instead of $S U(3)$ and four flavor degrees of freedom. Moreover, the quark mass at $m a=0.01$ is larger than its physical value. The situation can change, when one arrives at even smaller quark masses. We want to address this question in a future work.

The authors are grateful to V. I. Zakharov and V. G. Bornyakov for interesting and stimulating discussions. The simulations were performed at GPUs of supercomputer K100 and computers of the Berlin group. The work was supported by RFBR grants 14-02-01185-a, 13-02-01387-a, grant of the president of the RF MD-3215.2014.2 and grant of the FAIR-Russia Research Center.

\section{References}

[1] A. A. Belavin, A. M. Polyakov, A. S. Schwartz and Yu. S. Tyupkin. Pseudoparticle Solutions of the Yang-Mills Equations. Phys. Lett. B 59, 85 (1975).

[2] T. C. Kraan and P. van Baal. Periodic instantons with nontrivial holonomy. Nucl. Phys. B 533, 627-659 (1998), hep-th/9805168.

[3] K.-M. Lee and C.-H. Lu. SU(2) calorons and magnetic monopoles. Phys. Rev. D 58, 025011 (1998), hep-th/9802108.

[4] E. Witten. Current algebra theorems for the U(1) "Goldstone boson". Nucl. Phys. B 156, 269 (1979).

[5] G. Veneziano. U(1) Without Instantons. Nucl. Phys. B 159, 213 (1979).

[6] D. Kharzeev. Parity violation in hot QCD: Why it can happen, and how to look for it. Phys. Lett. B 633, 260 (2006), hep-ph / 0406125.

[7] K. Fukushima, D. E. Kharzeev and H. J. Warringa. The Chiral Magnetic Effect. Phys. Rev. D 78, 074033 (2008).

[8] N. S. Manton. Topology in the Weinberg-Salam Theory. Phys. Rev. D 28, 2019 (1983).

[9] F. R. Klinkhamer and N. S. Manton. A Saddle Point Solution in the Weinberg-Salam Theory. Phys. Rev. D 30, 2212 (1984).

[10] G. D. Moore. Do we understand the sphaleron rate? hep-ph/0009161.

[11] B. I. Abelev et al. [STAR Collaboration]. Azimuthal Charged-Particle Correlations and Possible Local Strong Parity Violation. Phys. Rev. Lett. 103, 251601 (2009), 0909.1739 [nucl-ex] .

[12] B. I. Abelev et al. [STAR Collaboration]. Observation of charge-dependent azimuthal correlations and possible local strong parity violation in heavy ion collisions. Phys. Rev. C 81, 054908 (2010),0909.1717 [nucl-ex].

[13] A. Yamamoto, Chiral magnetic effect in lattice QCD with a chiral chemical potential. Phys. Rev. Lett. 107, 031601 (2011), 1105.0385 [hep-lat] .

[14] A. Yamamoto, Lattice study of the chiral magnetic effect in a chirally imbalanced matter. Phys. Rev. D 84, 114504 (2011), 1111.4681 [hep-lat] . 
[15] A. A. Andrianov, D. Espriu and X. Planells. Chemical potentials and parity breaking: the Nambu-Jona-Lasinio model. Eur.Phys.J. C 74, 2776 (2014), 1310.4416 [hep-ph] .

[16] X. Planells, A. A. Andrianov, V. A. Andrianov and D. Espriu. An effective theory for QCD with an axial chemical potential. PoS QFTHEP 2013, 049 (2013), 1310.4434 [hep-ph] .

[17] K. Fukushima, M. Ruggieri and R. Gatto. Chiral magnetic effect in the PNJL model. Phys. Rev. D 81, 114031 (2010), 1003.0047 [hep-ph].

[18] M. N. Chernodub and A. S. Nedelin. Phase diagram of chirally imbalanced QCD matter. Phys. Rev. D 83, 105008 (2011), 1102.0188 [hep-ph] .

[19] R. Gatto and M. Ruggieri. Hot Quark Matter with an Axial Chemical Potential. Phys. Rev. D 85, 054013 (2012), 1110.4904 [hep-ph].

[20] E.-M. Ilgenfritz, M. Kalinowski, M. Müller-Preussker, B. Petersson and A. Schreiber. Two-color QCD with staggered fermions at finite temperature under the influence of a magnetic field. Phys. Rev. D 85, 114504 (2012), 1203.3360 [hep-lat] .

[21] E.-M. Ilgenfritz, M. Müller-Preussker, B. Petersson and A. Schreiber. Magnetic catalysis (and inverse catalysis) at finite temperature in two-color lattice QCD. Phys. Rev. D 89, 054512 (2014), $1310.7876[$ hep-lat $]$. 